November 6, 2001

Revised: January 31, 2002

CPT-2001/P.4260

\title{
Hadronic Light-By-Light Scattering Contribution to the Muon $g-2$ : An Effective Field Theory Approach
}

\author{
M. Knecht, A. Nyffeler, M. Perrottet, and E. de Rafael \\ Centre de Physique Théorique, CNRS-Luminy, Case 907 \\ F-13288 Marseille Cedex 9, France
}

\begin{abstract}
The hadronic light-by-light contribution to $a_{\mu}$, the anomalous magnetic moment of the muon, is discussed from the point of view of an effective low-energy theory. As an application, the coefficient of the leading logarithm arising from the two-loop graphs involving two anomalous vertices is computed, and found to be positive. This corresponds to a positive sign for the pion-pole contribution to the hadronic light-by-light correction to $a_{\mu}$, and to a sizeable reduction of the discrepancy between the present experimental value of $a_{\mu}$ and its theoretical counterpart in the standard model.
\end{abstract}

PACS: 13.40.Em, 12.20.Ds, 12.39.Fe, 14.60.Ef

Keywords: Muon magnetic moment, Effective field theory,

Renormalization group, Chiral symmetry. 



\title{
Hadronic Light-By-Light Scattering Contribution to the Muon $g-2$ :
}

\section{An Effective Field Theory Approach}

\author{
M. Knecht, A. Nyffeler, M. Perrottet, and E. de Rafael \\ Centre de Physique Théorique, CNRS-Luminy, Case 907, F-13288 Marseille Cedex 9, France
}

\begin{abstract}
The hadronic light-by-light contribution to $a_{\mu}$, the anomalous magnetic moment of the muon, is discussed from the point of view of an effective low-energy theory. As an application, the coefficient of the leading logarithm arising from the two-loop graphs involving two anomalous vertices is computed, and found to be positive. This corresponds to a positive sign for the pion-pole contribution to the hadronic light-by-light correction to $a_{\mu}$, and to a sizeable reduction of the discrepancy between the present experimental value of $a_{\mu}$ and its theoretical counterpart in the standard model.
\end{abstract}

The Brookhaven E821 experiment has recently measured [1] the anomalous magnetic moment $a_{\mu}$ of the muon with a precision of $\pm 16 \times 10^{-10}$, improving by a factor of 6 on the previous measurement at CERN [2]. As the full set of data will be analyzed, the experimental error bars are expected to decrease further, by at least an additional factor of 3 . The discrepancy between the experimental value of Ref. [1] and certain theoretical estimates can be as large as 2.6 $\sigma$. Many possibilities to explain this difference through the introduction of new, beyond the standard model, degrees of freedom have therefore been considered. It is however important to keep in mind that the theoretical estimates of $a_{\mu}$ include several contributions involving the nonperturbative hadronic sector of the standard model. In this Letter, we shall focus on one of these contributions, the so-called hadronic lightby-light scattering, which arises from the lowest order contribution, in the fine-structure constant $\alpha$, to the matrix element ( $e$ stands for the electron charge)

$$
\left\langle\mu^{-}\left(p^{\prime}\right)\left|(i e) j_{\rho}(0)\right| \mu^{-}(p)\right\rangle \equiv(-i e) \overline{\mathrm{u}}\left(p^{\prime}\right) \widehat{\Gamma}_{\rho}\left(p^{\prime}, p\right) \mathrm{u}(p)
$$

of the conserved light quark electromagnetic current, $j_{\rho}=\left(2 \bar{u} \gamma_{\rho} u-\bar{d} \gamma_{\rho} d-\bar{s} \gamma_{\rho} s\right) / 3$. This matrix element is given by a two-loop integral involving the connected fourth rank hadronic vacuum polarization tensor $\Pi_{\mu \nu \lambda \rho}\left(q_{1}, q_{2}, q_{3}\right)$. The corresponding contribution $a_{\mu}^{\mathrm{LbyL} ; \mathrm{had}}$ to $a_{\mu}$ is equal to

$$
\lim _{p^{\prime}-p \rightarrow 0} \operatorname{tr}\left[(\not p+m) \Lambda_{\rho}^{(2)}\left(\not p^{\prime}+m\right) \widehat{\Gamma}^{\rho}\left(p^{\prime}, p\right)\right]
$$

with $\left(k=p^{\prime}-p\right)$

$$
\Lambda_{\rho}^{(2)}=\frac{m^{2}}{k^{2}\left(4 m^{2}-k^{2}\right)}\left[\gamma_{\rho}+\frac{k^{2}+2 m^{2}}{m\left(k^{2}-4 m^{2}\right)}\left(p^{\prime}+p\right)_{\rho}\right] .
$$

This hadronic light-by-light correction to $a_{\mu}$ has been studied by several authors in the past [3 8]. Its value, and even its sign, has suffered several changes, but the latest evaluations came to values that agreed within the quoted theoretical error bars and that were negative. In particular, there exists a well defined contribution to $a_{\mu}^{\mathrm{LbyL} ; \mathrm{had}}$, denoted by $a_{\mu}^{\mathrm{LbyL} ; \pi^{0}}$, that arises upon restricting $\Pi_{\mu \nu \lambda \rho}\left(q_{1}, q_{2}, q_{3}\right)$ to its reducible one-pion exchange component and which represents about $70 \%$ of the total value of $a_{\mu}^{\text {LbyL; had }}$. Very recently, two of us, reconsidering this evaluation of $a_{\mu}^{\mathrm{Lby} ; ;} \pi^{0}$, found a positive result [9], but which, in absolute value, agreed with the previous numerical values whenever comparison was possible. Resolving this sign ambiguity certainly represents a major issue, since the result of [9] reduces the theory vs experiment discrepancy to less than $1.5 \sigma$. This Letter aims at providing an argument in favor of a positive sign for $a_{\mu}^{\mathrm{LbyL} ; \pi^{0}}$ which, to a large extent, does not rely on the methodology followed in Ref. 99. The offshell pion-photon-photon form factor $\mathcal{F}_{\pi^{0} \gamma^{*} \gamma^{*}}$ constitutes an important ingredient for the evaluation of $a_{\mu}^{\mathrm{Lby} ; \pi^{0}}$. For a constant form factor, fixed by the Wess-ZuminoWitten (WZW) term of QCD [10], which reproduces the Adler [11], Bell-Jackiw [12] anomaly, the corresponding two-loop integral for $a_{\mu}^{\text {Lby ; } \pi^{0}}$ diverges like $(\alpha / \pi)^{3} \mathcal{C} \ln ^{2} \Lambda$, where $\Lambda$ stands for an ultraviolet cutoff 13 . We shall be interested in the determination of the coefficient $\mathcal{C}$ of this log-squared divergence, so that we may then compare it to the value that can be extracted from the analysis of Ref. [9]. In order to achieve this goal, we shall use a renormalization group argument, along the lines discussed in Ref. [14, within the framework of the effective low-energy field theory of the standard model. Before doing so, we first describe the relevant features of this effective theory.

The low-energy degrees of freedom of the standard model involve the pseudoscalar mesons, the light leptons, and the photon. The interactions between these degrees of freedom are constrained by the symmetries of the standard model, like chiral symmetry or $U(1)$ gauge invariance. This effective theory will provide a good description of physical processes as long as the energy scales involved are much lower than a typical hadronic scale $\Lambda_{H} \sim 1 \mathrm{GeV}$. Since the leptons obey a first order equation of motion, the chiral counting has to be suit- 
ably adapted. The situation differs from the case of chiral perturbation theory in the presence of baryons due to the fact that the mass $m$ of the light leptons does not introduce a scale of the order of $\Lambda_{H}$. Rather, the counting $m \sim \mathcal{O}(\mathrm{p})$ arises quite naturally. Furthermore, we shall also count fermion bilinears as quantities of order $\mathrm{p}$, as is the case in the sector of the effective theory which describes the semileptonic decays of the pseudoscalar mesons [15] (see also Ref. 16] for a discussion of this aspect in a different context). Finally, the electric charge is also counted as a quantity of order $\mathrm{p}[17$. The lowest order term in this extended chiral expansion then starts at order $\mathrm{p}^{2}$, with (for the notation, see Refs. 18, 19,17 ):

$$
\begin{aligned}
\mathcal{L}^{(2)} & =-\frac{1}{4} F_{\mu \nu} F^{\mu \nu}+\bar{\psi}(i \not D-m) \psi+e^{2} C\left\langle Q U^{+} Q U\right\rangle \\
& +\frac{F_{0}^{2}}{4}\left(\left\langle d^{\mu} U^{+} d_{\mu} U\right\rangle+2 B_{0}\left\langle\mathcal{M}^{+} U+U^{+} \mathcal{M}\right\rangle\right) .
\end{aligned}
$$

This lowest order Lagrangian involves the Maxwell Lagrangian for the photon, the tree-level minimal coupling of leptons and photons, as well as the lowest order chiral Lagrangian for the mesons, together with their couplings to the photons. It gives the (finite) lowest order, $\mathcal{O}\left(\mathrm{p}^{6}\right)$, contribution to $a_{\mu}^{\mathrm{LbyL} ; \text { had }}$ obtained upon restricting $\Pi_{\mu \nu \lambda \rho}\left(q_{1}, q_{2}, q_{3}\right)$ to its lowest order approximation, consisting of a charged pion loop with pointlike electromagnetic vertices. At $\mathcal{O}\left(\mathrm{p}^{4}\right)$ in the Lagrangian, we find the usual counterterms involving the low-energy constants $L_{i}$ [18], $K_{i}$ [17,20] and $X_{i}$ [15]. These counterterms absorb the divergences due to loops with virtual pions, photons, and leptons. For instance, $L_{9}$ is needed in order to make the slope of the pion form factor, generated by a charged pion loop, finite. Note that the pion loop contribution to $\Pi_{\mu \nu \lambda \rho}\left(q_{1}, q_{2}, q_{3}\right)$ with these nonpointlike vertices, gives a next-to-leading, $\mathcal{O}\left(\mathrm{p}^{8}\right)$, correction to $a_{\mu}^{\mathrm{LbyL} \text {, had }}$ which is no longer finite, and thus requires a corresponding counterterm from $\mathcal{L}^{(10)}$. In addition, we also encounter in $\mathcal{L}^{(4)}$ the counterterms which result from the divergent loop graphs involving virtual leptons and photons, such as the $\mathcal{O}(\alpha)$ renormalizations of $e, m$ and of the lepton wave function. These will play no role in the present discussion. Finally, the WZW Lagrangian of the odd intrinsic parity sector also occurs in $\mathcal{L}^{(4)}$. For our present purpose, we need only to retain a piece of the latter (our conventions are as follows: $\left.\gamma_{5}=i \gamma^{0} \gamma^{1} \gamma^{2} \gamma^{3}, \varepsilon_{0123}=+1\right)$

$$
\begin{aligned}
\mathcal{L}^{(4)}= & \frac{i e^{2} N_{C}}{24 \pi^{2}} \varepsilon_{\mu \nu \alpha \beta} \partial^{\mu} A^{\nu} A^{\alpha}\left\langle Q^{2} \partial^{\beta} U U^{+}+Q^{2} U^{+} \partial^{\beta} U\right. \\
& \left.-\frac{1}{2} Q U Q \partial^{\beta} U^{+}+\frac{1}{2} Q U^{+} Q \partial^{\beta} U\right\rangle+\ldots \\
= & -\frac{\alpha N_{C}}{12 \pi F_{0}} \varepsilon_{\mu \nu \alpha \beta} F^{\mu \nu} A^{\alpha} \partial^{\beta} \pi^{0}+\ldots
\end{aligned}
$$

This term is responsible for the lowest order, and divergent, contribution to the pion-pole correction $a_{\mu}^{\mathrm{LbyL} ; \pi^{0}}$, which thus starts at order $\mathrm{p}^{8}$ and corresponds to the diagrams (a), (b), and (c) of Fig. 1. At order $\mathrm{p}^{6}$ in the effective field theory, we encounter, for instance, divergent loops involving the particular WZW vertex from $\mathcal{L}^{(4)}$ shown in Eq. (位) and a virtual fermion line, like precisely the triangular subgraphs in the two first graphs of Fig. 1. These divergences have to be canceled by an appropriate set of counterterms which, according to Ref. [21], reads

$$
\begin{aligned}
\mathcal{L}^{(6)}= & \frac{3 i \alpha^{2}}{32 \pi^{2}} \bar{\psi} \gamma_{\mu} \gamma_{5} \psi\left\{\chi_{1}\left\langle Q^{2}\left(U^{+} d^{\mu} U+d^{\mu} U U^{+}\right)\right\rangle\right. \\
& \left.+\chi_{2}\left\langle Q U^{+} Q d^{\mu} U-Q d^{\mu} U^{+} Q U\right\rangle\right\}+\ldots
\end{aligned}
$$

Here also, there may be other terms, indicated by the ellipsis, but which are of no relevance for the discussion concerning $a_{\mu}^{\mathrm{LbyL} ; \pi^{0}}$. Under renormalization, the terms we have shown absorb the divergence of the triangular subgraphs in the diagrams (a) and (b) of Fig. 1. They therefore also contribute to $a_{\mu}^{\mathrm{Lby} L ;} \pi^{0}$ at order $\mathrm{p}^{8}$, through the diagrams (d) and (e) of Fig. 1. We shall come back to this important issue below. Let us first conclude this brief description of the effective theory by mentioning that the graphs (a) and (b) also have an overall divergence, which is absorbed by an appropriate set of counterterms in $\mathcal{L}^{(10)}$, the effective Lagrangian at the $\mathcal{O}\left(\mathrm{p}^{10}\right)$ level. The corresponding tree-level contribution to $a_{\mu}^{\mathrm{Lby} ;} ; \pi^{0}$ is represented by the diagram (f) in Fig. 1. The detailed structure of $\mathcal{L}^{(10)}$ will, however, not be needed.

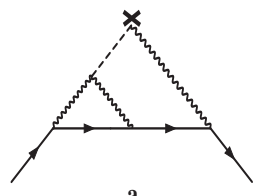

a

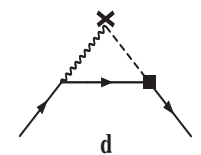

d

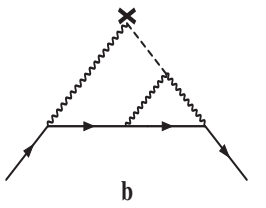

b

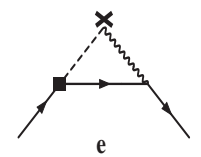

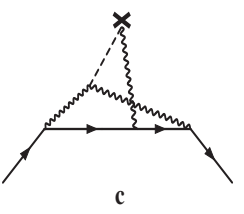

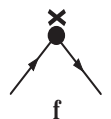

FIG. 1. The graphs contributing to $a_{\mu}^{\mathrm{LbyL} ; \pi^{0}}$ at lowest order in the effective field theory. The two-loop graphs (a), (b), and (c) contain two WZW vertices from (4). The one-loop graphs (d) and (e) have an insertion of one vertex $(\mathbf{\square})$ of $\mathcal{L}^{(6)}$, Eq. ([). Finally, the tree-level graph (f) stems from the $\mathcal{O}\left(\mathrm{p}^{10}\right)$ counterterms. Note that the diagram (c) is actually finite.

We next return to the original purpose of this Letter, the determination of the two-loop leading logarithm arising from the sum of the diagrams (a) and (b) of Fig. 1. Since the diagram (c) is finite, it is of no relevance for the present discussion. In the context of a renormalizable field theory, such a question would be most naturally addressed by means of the renormalization group equations. As stressed by Weinberg [14], the renormalization group remains a useful concept even in the case of a nonrenormalizable local effective field theory. Within the 
effective theory framework, the expression of the renormalized contribution to $a_{\mu}$ arising from the graphs of Fig. 1 takes the general form

$$
a_{\mu}^{\mathrm{LbyL} ; \pi^{0}}=H\left(\frac{m}{\mu}\right)+\chi(\mu) J\left(\frac{m}{\mu}\right)+\kappa(\mu) .
$$

Here $\mu$ denotes the arbitrary subtraction scale introduced by the renormalization procedure. The contributions of the two-loop graphs (a), (b), and (c) of Fig. 1 are given by the first term in this expression. The next term describes the contributions from the one-loop graphs (d) and (e), with an insertion of the renormalized $\mathcal{O}\left(\mathrm{p}^{6}\right)$ counterterm $\chi \equiv(-1 / 4)\left(\chi_{1}+\chi_{2}\right)$, while the last term, $\kappa(\mu)$, collectively stands for the renormalized tree-level contributions from the $\mathcal{O}\left(\mathrm{p}^{10}\right)$ effective Lagrangian. The dependence on the subtraction scale $\mu$ in the two- and one-loop functions reads

$$
\begin{aligned}
& H\left(\frac{m}{\mu}\right)=\sum_{p=0,1,2} h_{p} \ln ^{p}\left(\frac{m}{\mu}\right) \\
& J\left(\frac{m}{\mu}\right)=\sum_{q=0,1} j_{q} \ln ^{q}\left(\frac{m}{\mu}\right) .
\end{aligned}
$$

The (dimensionless) coefficients $h_{p}$ and $j_{q}$ do not depend on $\mu$, but are functions of the ratios $M_{\pi^{0}} / m$ and $F_{\pi} / m$. They therefore satisfy $\mathcal{D} h_{p}=0, \mathcal{D} j_{q}=0$, where

$$
\mathcal{D} \equiv m \frac{\partial}{\partial m}+M_{\pi^{0}} \frac{\partial}{\partial M_{\pi^{0}}}+F_{\pi} \frac{\partial}{\partial F_{\pi}}
$$

Being a physical quantity, $a_{\mu}^{\mathrm{LbyL} ; \pi^{0}}$ obeys the condition $\mu\left(d a_{\mu}^{\mathrm{LbyL} ; \pi^{0}} / d \mu\right)=0$, which gives

$$
\begin{aligned}
& \ln \left(\frac{m}{\mu}\right)\left[2 h_{2}-\gamma_{\chi} j_{1}\right]+h_{1}-\gamma_{\chi} j_{0}+\chi(\mu) j_{1} \\
& -\mu \frac{d \kappa(\mu)}{d \mu}=0
\end{aligned}
$$

where we have introduced $\gamma_{\chi}=\mu[d \chi(\mu) / d \mu]$. Acting with the operator $\mathcal{D}$ on this equation and using the fact that $\mathcal{D} \chi(\mu)=0, \mathcal{D} \kappa(\mu)=0$, one finds that the term proportional to $\ln (m / \mu)$ has to vanish separately, i.e.,

$$
h_{2}\left(\frac{M_{\pi^{0}}}{m}, \frac{F_{\pi}}{m}\right)=\frac{1}{2} \gamma_{\chi} j_{1}\left(\frac{M_{\pi^{0}}}{m}, \frac{F_{\pi}}{m}\right),
$$

and that

$$
\kappa(\mu)=\frac{1}{2} \gamma_{\chi} j_{1}\left(\frac{M_{\pi^{0}}}{m}, \frac{F_{\pi}}{m}\right) \ln ^{2}\left(\frac{\mu}{\mu_{0}}\right)+\cdots .
$$

Therefore, in order to compute $h_{2} \equiv(\alpha / \pi)^{3} \mathcal{C}$, we have to extract the dependence on $\ln (m / \mu)$ from the one-loop graphs of Fig. 1. This is our next step.

The sum of the two graphs (d) and (e) of Fig. 1 reads

$$
\begin{aligned}
& (-i e) \overline{\mathrm{u}}\left(p^{\prime}\right) \widehat{\Gamma}_{\rho}^{(\mathrm{d})+(\mathrm{e})}\left(p^{\prime}, p\right) \mathrm{u}(p)=\left(\frac{\alpha^{2}}{4 \pi^{2} F_{\pi}}\right)\left(\frac{i \alpha N_{C}}{3 \pi F_{\pi}}\right) \\
& \times \chi(\mu) \int \frac{d^{4} q}{(2 \pi)^{4}} \frac{-i}{(k-q)^{2}} \frac{i}{q^{2}-M_{\pi^{0}}^{2}} \varepsilon_{\rho \lambda \alpha \beta} q^{\alpha} k^{\beta} \\
& \times \overline{\mathrm{u}}\left(p^{\prime}\right)\left[(-i e) \gamma^{\lambda} \frac{i}{\not p+\not 1-m} \not \gamma_{5}\right. \\
& \left.+\not q \gamma_{5} \frac{i}{\not p^{\prime}-\not q-m}(-i e) \gamma^{\lambda}\right] \mathrm{u}(p) .
\end{aligned}
$$

This integral diverges logarithmically. Introducing a cutoff $\Lambda$, the corresponding contribution to $a_{\mu}$ is obtained upon using (2). The Dirac trace can easily be performed, leading to the result $\left.a_{\mu}^{\mathrm{LbyL} ; \pi^{0}}\right|_{(\mathrm{d})+(\mathrm{e})}=j_{1} \ln (m / \Lambda)+$ finite, with

$$
j_{1}=\frac{N_{C}}{24 \pi^{2}}\left(\frac{\alpha}{\pi}\right)^{3}\left(\frac{m}{F_{\pi}}\right)^{2}
$$

It is interesting to note that the result for $j_{1}$ can also be obtained from the calculation of the coefficient of the $\ln \left(M_{Z}^{2} / m^{2}\right)$ term in the two-loop electroweak contribution to $a_{\mu}$; see Ref. [22]. Next, we recall that the scale dependence of the constant $\chi(\mu)$ is already known [21,23. Indeed, the same combination of $\chi_{1}$ and $\chi_{2}$ arises in the $\mathcal{O}\left(\alpha^{2}\right)$ contribution to the $\pi^{0} \rightarrow e^{+} e^{-}$amplitude, together with the divergent one-loop graph, generated by the WZW vertex, and involving two virtual photons and one fermion line [the on-shell restriction of the triangular subgraphs in diagrams (a) and (b)]. The result reads $\gamma_{\chi}=N_{C}$, and thus with Eqs. (6) and (10) we obtain

$$
a_{\mu}^{\mathrm{LbyL} ; \pi^{0}}=\left(\frac{\alpha}{\pi}\right)^{3}\left[\mathcal{C} \ln ^{2}\left(\frac{m}{\mu_{0}}\right)+\mathcal{O}\left[\ln \left(m / \mu_{0}\right)\right]\right],
$$

where

$$
\mathcal{C}=+3\left(\frac{N_{C}}{12 \pi}\right)^{2}\left(\frac{m}{F_{\pi}}\right)^{2}
$$

For $F_{\pi}=92.4 \mathrm{MeV}, m=105.66 \mathrm{MeV}$ and $N_{C}=3$, this gives $\mathcal{C}=0.0248$. We shall now discuss some consequences of this result as far as the $\operatorname{sign}$ of $a_{\mu}^{\mathrm{LbyL} ; \pi^{0}}$ obtained in Ref. [9] is concerned.

Our analysis thus tells us that the sum of the graphs (a) and (b) of Fig. 1 not only diverges, but behaves like $(\alpha / \pi)^{3} \mathcal{C} \ln ^{2} \Lambda$ as the ultraviolet cutoff $\Lambda$ is sent to infinity. Actual calculations of $a_{\mu}^{\mathrm{LbyL} ; \pi^{0}}$ [which, in the framework of the effective theory, amount to estimates of $\chi(\mu)$ and of $\kappa(\mu)$ ] replace the pointlike WZW vertex by a form factor $\mathcal{F}_{\pi^{0} \gamma^{*} \gamma^{*}}$ which regularizes the ultraviolet behavior. The models of $\mathcal{F}_{\pi^{0} \gamma^{*} \gamma^{*}}$ that are introduced for this purpose in one way or another involve a hadronic scale $\Lambda_{H}$, typically the $\rho$-meson mass $M_{V}$ (or $\Lambda_{H} \sim 2 m_{Q}$, with a constituent quark mass $\left.m_{Q}.\right)$. As $M_{V} \rightarrow \infty$, the form factor becomes constant, and one therefore has to recover the same behavior, $a_{\mu}^{\mathrm{LbyL} ; \pi^{0}} \sim(\alpha / \pi)^{3} \mathcal{C} \ln ^{2} M_{V}$. To the best of our 
knowledge, this aspect of $a_{\mu}^{\mathrm{LbyL} ; \pi^{0}}$ has not been discussed in detail before. In particular, we know of no previous determination of the constant $\mathcal{C}$. There is a reference to the $\ln ^{2} \Lambda$ behavior in [13], which, in our notation, states that $\left.a_{\mu}^{\mathrm{LbyL} ; \pi^{0}}\right|_{W Z W} \sim(\alpha / \pi)^{3}\left(m / 4 \pi F_{\pi}\right)^{2}\left(\ln ^{2} \Lambda\right) / 2$, but it is not clear from the context of Ref. [13] whether the sign was meant to be part of the estimate or not.

In Ref. [9], $a_{\mu}^{\mathrm{LbyL} ; \pi^{0}}$ was expressed, for a certain class of form factors, in terms of a two-dimensional integral representation of the form (we omit here the small contribution from the finite diagram (c) in Fig. 1, which plays no role in the present discussion)

$$
\begin{array}{r}
a_{\mu}^{\mathrm{LbyL} ; \pi^{0}}=\left(\frac{\alpha}{\pi}\right)^{3} \int_{0}^{\infty} d Q_{1} \int_{0}^{\infty} d Q_{2}\left[f\left(M_{V}, Q_{1}, Q_{2}\right) w_{f}\left(Q_{1}, Q_{2}\right)\right. \\
\left.+g\left(M_{V}, Q_{1}, Q_{2}\right) w_{g}\left(M_{V}, Q_{1}, Q_{2}\right)\right] .
\end{array}
$$

The functions $f\left(M_{V}, Q_{1}, Q_{2}\right)$ and $g\left(M_{V}, Q_{1}, Q_{2}\right)$ depend quadratically on $\mathcal{F}_{\pi^{0} \gamma^{*} \gamma^{*}}$. The weight functions $w_{f}\left(Q_{1}, Q_{2}\right)$ and $w_{g}\left(M_{V}, Q_{1}, Q_{2}\right)$ are both positive and peaked around $Q_{1} \sim Q_{2} \sim 0.5 \mathrm{GeV}$. In the case of the vector meson dominance (VMD) form factor, $f^{V M D}\left(M_{V}, Q_{1}, Q_{2}\right)$ vanishes, and only the term involving $w_{g}\left(M_{V}, Q_{1}, Q_{2}\right)$ contributes. The corresponding value obtained in [9] for $\left.a_{\mu}^{\mathrm{Lby} ; \pi^{0}}\right|_{V M D}$ upon numerical evaluation of the integral (16) differs only by its overall sign from the results obtained by previous authors [5, 6]. Now, in Ref. [9], it has been shown that (up to $\sim \ln M_{V}$ or constant terms)

$$
\begin{aligned}
& \lim _{M_{V} \rightarrow \infty} \int_{0}^{\infty} d Q_{1} \int_{0}^{\infty} d Q_{2} g^{V M D}\left(M_{V}, Q_{1}, Q_{2}\right) w_{g}\left(M_{V}, Q_{1}, Q_{2}\right) \\
& \quad=\mathcal{C} \ln ^{2} M_{V}
\end{aligned}
$$

with a constant $\mathcal{C}$ that numerically agrees with the result of Eq. (15), including its sign. A global sign error in the second contribution to $(16)$ is thus excluded. On the other hand, for a constant form factor, normalized by the WZW term, $f^{W Z W}\left(M_{V}, Q_{1}, Q_{2}\right)=\left[N_{C} /\left(12 \pi^{2} F_{\pi}\right)\right]^{2}$ and $g^{W Z W}\left(M_{V}, Q_{1}, Q_{2}\right)$ vanishes. In that case, the integral involving $w_{f}\left(Q_{1}, Q_{2}\right)$ diverges. In Ref. [9], it was shown that this divergence is of the form (up to $\sim \ln \Lambda$ or constant terms)

$$
\begin{aligned}
& \lim _{\Lambda \rightarrow \infty} \int_{0}^{\Lambda} d Q_{1} \int_{0}^{\Lambda} d Q_{2} f^{W Z W}\left(M_{V}, Q_{1}, Q_{2}\right) w_{f}\left(Q_{1}, Q_{2}\right) \\
& \quad=\mathcal{C} \ln ^{2} \Lambda
\end{aligned}
$$

where, again, the constant $\mathcal{C}$ was found [9] to be positive and in agreement with Eq. (15). We thus conclude that the overall sign of the first term in Eq. (16) also has to be correct.

Therefore, we expect that $a_{\mu}^{\mathrm{LbyL} ; \pi^{0}}$ is a positive quantity, at least for the VMD type of parametrizations of the form factor $\mathcal{F}_{\pi^{0} \gamma^{*} \gamma^{*}}$. This result was also found to hold for a wider class of form factors studied in Ref. [9].
The implications of these observations for the comparison between theory and experiment as far as the anomalous magnetic moment of the muon is concerned have already been discussed in Ref. [9]. In particular, the difference between the present experimental value of $a_{\mu}$ [1] and the standard model is reduced from $2.6 \sigma$ to $1.5 \sigma$. The extension of the present effective field theory analysis to the other contributions from hadronic light-by-light scattering to the muon $g-2$ will be discussed elsewhere.

This work has been supported in part by Schweizerischer Nationalfonds and by TMR, EC Contract No. ERBFMRX-CT980169 (EURODAPHNE).

[1] Muon $g-2$ Collaboration, H. N. Brown et al., Phys. Rev. Lett. 86, 2227 (2001).

[2] J. Bailey et al., Phys. Lett. 68B, 191 (1977).

[3] J. Calmet et al., Phys. Lett. 61B, 283 (1976); Rev. Mod. Phys. 49, 21 (1977).

[4] T. Kinoshita, B. Nižić and Y. Okamoto, Phys. Rev. Lett. 52, 717 (1984); Phys. Rev. D 31, 2108 (1985); Y. Okamoto, Ph.D. thesis, Cornell University, 1984 (unpublished).

[5] M. Hayakawa, T. Kinoshita and A. I. Sanda, Phys. Rev. Lett. 75, 790 (1995); Phys. Rev. D 54, 3137 (1996); M. Hayakawa and T. Kinoshita, Phys. Rev. D 57, 465 (1998).

[6] J. Bijnens, E. Pallante and J. Prades, Phys. Rev. Lett. 75, 1447 (1995); 75, 3781(E) (1995); Nucl. Phys. B474, 379 (1996).

[7] J. Bijnens and F. Persson, hep-ph/0106130.

[8] E. Bartoš et al., hep-ph/0106084 v2.

[9] M. Knecht and A. Nyffeler, Marseille Report No. CPT2001/P.4253, hep-ph/0111058 [Phys. Rev. D (to be published)].

[10] J. Wess and B. Zumino, Phys. Lett. 37B, 95 (1971); E. Witten, Nucl. Phys. B223, 422 (1983).

[11] S.L. Adler, Phys. Rev. 177, 2426 (1969).

[12] J.S. Bell and R. Jackiw, Nuovo Cim. A 60, 47 (1969).

[13] K. Melnikov, Int. J. Mod. Phys. A 16, 4591 (2001).

[14] S. Weinberg, Physica (Amsterdam) 96A, 327 (1979).

[15] M. Knecht et al., Eur. Phys. J. C 12, 469 (2000).

[16] A. Nyffeler and A. Schenk, Phys. Rev. D 62, 113006 (2000).

[17] R. Urech, Nucl. Phys. B433, 234 (1995).

[18] J. Gasser and H. Leutwyler, Nucl. Phys. B250, 465 (1985).

[19] G. Ecker et al., Nucl. Phys. B321, 311 (1989).

[20] H. Neufeld and H. Rupertsberger, Z. Phys. C 68, 91 (1995); 71, 131 (1996).

[21] M.J. Savage, M. Luke and M.B. Wise, Phys. Lett. B 291, 481 (1992).

[22] S. Peris, M. Perrottet and E. de Rafael, Phys. Lett. B 355, 523 (1995).

[23] Ll. Ametller, A. Bramon and E. Massó, Phys. Rev. D 48, 3388 (1993); D. Gómez Dumm and A. Pich, Phys. Rev. Lett. 80, 4633 (1998); M. Knecht et al., Phys. Rev. Lett. 83, 5230 (1999). 